\title{
Review of the efficacy of infrared thermography for screening infectious diseases with applications to COVID-19
}

\author{
Kosar Khaksari $\odot,{ }^{\mathrm{a}, \dagger}{ }^{\dagger}$ Thien Nguyen $\odot,{ }^{\mathrm{a}, \dagger}$ Brian Hill, ${ }^{\mathrm{a}}$ Timothy Quang, ${ }^{\mathrm{a}}$ \\ John Perreault, ${ }^{a}$ Viswanath Gorti $\odot,{ }^{a}$ Ravi Malpani, ${ }^{a}$ Emily Blick, ${ }^{a}$ \\ Tomás González Cano, ${ }^{\mathrm{a}}$ Babak Shadgan, ${ }^{\mathrm{b}}$ and Amir H. Gandjbakhche ${ }^{\mathrm{a}, *}$ \\ ${ }^{a}$ National Institutes of Health, Eunice Kennedy Shrive National Institute of Child Health \\ and Human Development, Bethesda, Maryland, United States \\ ${ }^{b}$ University of British Columbia, Vancouver, British Columbia, Canada
}

\begin{abstract}
Purpose: The recent coronavirus disease 2019 (COVID-19) pandemic, which spread across the globe in a very short period of time, revealed that the transmission control of disease is a crucial step to prevent an outbreak and effective screening for viral infectious diseases is necessary. Since the severe acute respiratory syndrome (SARS) outbreak in 2003, infrared thermography (IRT) has been considered a gold standard method for screening febrile individuals at the time of pandemics. The objective of this review is to evaluate the efficacy of IRT for screening infectious diseases with specific applications to COVID-19.
\end{abstract}

Approach: A literature review was performed in Google Scholar, PubMed, and ScienceDirect to search for studies evaluating IRT screening from 2002 to present using relevant keywords. Additional literature searches were done to evaluate IRT in comparison to traditional core body temperature measurements and assess the benefits of measuring additional vital signs for infectious disease screening.

Results: Studies have reported on the unreliability of IRT due to poor sensitivity and specificity in detecting true core body temperature and its inability to identify asymptomatic carriers. Airport mass screening using IRT was conducted during occurrences of SARS, Dengue, Swine Flu, and Ebola with reported sensitivities as low as zero. Other studies reported that screening other vital signs such as heart and respiratory rates can lead to more robust methods for early infection detection.

Conclusions: Studies evaluating IRT showed varied results in its efficacy for screening infectious diseases. This suggests the need to assess additional physiological parameters to increase the sensitivity and specificity of non-invasive biosensors.

(C) The Authors. Published by SPIE under a Creative Commons Attribution 4.0 Unported License. Distribution or reproduction of this work in whole or in part requires full attribution of the original publication, including its DOI. [DOI: 10.1117/1.JMI.8.S1.010901]

Keywords: infrared thermography; thermal camera; core body temperature; fever screening; coronavirus; infectious disease.

Paper 20162VSSR received Jun. 22, 2020; accepted for publication Mar. 4, 2021; published online Mar. 25, 2021.

\section{Introduction}

This paper reviews and summarizes existing information on the sensitivity, specificity, positive predictive value (PPV), and negative predictive value (NPV) of infrared thermography (IRT) utilized in screening for fever as well as the incidence of respiratory infectious diseases during a pandemic. The screening scale ranges from a small, well-controlled laboratory to a massive,

\footnotetext{
*Address all correspondence to Amir H. Gandjbakhche, gandjbaa@mail.nih.gov

${ }^{\dagger}$ These authors contributed equally.
} 
uncontrolled airport. In addition, we discuss the advantages and limitations of IRT in detecting infection and suggest solutions for these limitations. Finally, we present options for the use of IRT toward the detection, diagnosis, and monitoring of coronavirus disease 2019 (COVID-19) during this ongoing pandemic.

\subsection{Infectious Diseases, Symptoms, Early Screening}

Infectious diseases are caused by pathogenic microorganisms such as bacteria, viruses, parasites, or fungi that are spread, directly or indirectly, between individuals. ${ }^{1}$ The known pandemics throughout history have been related to bacteria and viruses (Fig. 1). ${ }^{2,3}$ Currently, the worldwide outbreak of the novel COVID-19 poses a significant threat to global health. The virus causing COVID-19, severe acute respiratory syndrome coronavirus 2 (SARS-CoV-2), is in the coronavirus family that caused the outbreaks of severe acute respiratory syndrome (SARS) and Middle East respiratory syndrome (MERS). ${ }^{4}$ As of June 15, 2020, SARS-CoV-2 has infected 7,963,453 people worldwide, killing a total of 434,388 , numbers that will increase as the virus continues to spread. ${ }^{5,6}$

Symptoms of infectious diseases vary depending on the bacterial or viral agent; however, there are two common symptoms reported in many diseases. These are a fever and an increased antibody count in the blood. ${ }^{7}$ The diseases caused by the coronavirus family appear to result in similar symptoms, including fever, headache, dry cough, shortness of breath, gastrointestinal distress, and pneumonia (Table 1).

Early screening and diagnosis of infectious diseases can prevent or reduce the spread of the diseases, improve the effectiveness of treatment options, and reduce health care costs. ${ }^{8}$ Healthcare workers who are repeatedly exposed to the disease are at high risk of getting an infection. ${ }^{9,10}$ The elderly, those possessing pre-existing health conditions such as chronic lung, heart, liver, kidney diseases, obesity, and diabetes, and those who are immunocompromised are

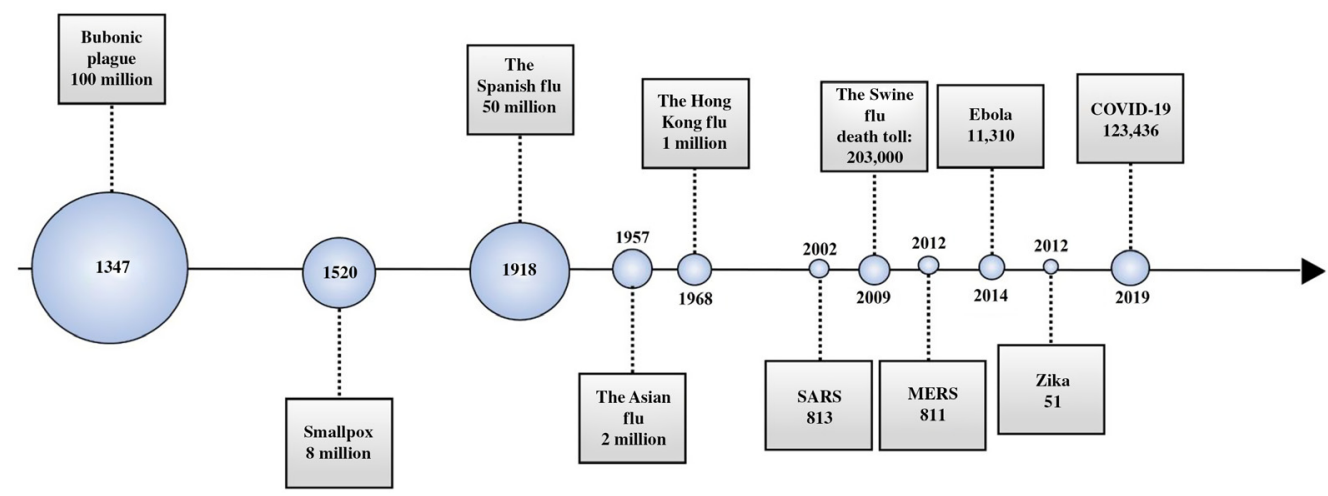

Fig. 1 Timeline overviewing history and impact of major pandemics and their death tolls. ${ }^{2,3}$

Table 1 Infectious diseases caused by coronavirus species.

\begin{tabular}{|c|c|c|c|c|c|c|}
\hline Virus & Disease & $\begin{array}{c}\text { Incubation } \\
\text { period (days) }\end{array}$ & Symptoms & $\begin{array}{l}\text { Infected } \\
\text { people }\end{array}$ & Deaths & $\begin{array}{l}\text { Fatality } \\
\text { rate (\%) }\end{array}$ \\
\hline SARS-CoV & SARS & $\begin{array}{c}4.7(95 \% \mathrm{Cl} \\
4.3 \text { to } 5.1)\end{array}$ & $\begin{array}{l}\text { Fever, headache, dry } \\
\text { cough, shortness of } \\
\text { breath, pneumonia, }\end{array}$ & 8437 & 813 & 10 \\
\hline MERS-CoV & MERS & $\begin{array}{c}5.2(95 \% \mathrm{Cl} \\
5.0 \text { to } 6.5)\end{array}$ & and gastrointestinal & 2442 & 811 & 35 \\
\hline SARS-CoV-2 & COVID-19 & $\begin{array}{c}4.9(95 \% \mathrm{Cl} \\
4.4 \text { to } 5.5)\end{array}$ & & $7,963,453^{a}$ & $434,388^{a}$ & \\
\hline
\end{tabular}

${ }^{\mathrm{a}}$ Data collected on June 15,$2020 ;{ }^{5}$ numbers continuing to rise. 
at higher risk of a serious illness or life threatening infection. ${ }^{11}$ Considering the large groups of at-risk individuals, screening and early detection/diagnosis of the disease are crucial.

\subsection{Fever Screening}

Infrared thermometers or thermal cameras have been used extensively to screen febrile patients and travelers at the time of pandemic for non-contact and rapid monitoring of body temperature. Mass fever screenings have been performed in different places such as airports, ${ }^{12,13}$ ports (seaports) ${ }^{14,15}$ border (ground) crossings ${ }^{15,16}$ and other public places such as hospital entrances. There is no strong evidence of the effectiveness of port and border crossing mass screening in delaying local transmission. ${ }^{17}$ Airport screening for fever was common at the time of pandemics. ${ }^{17-19}$ The intention was to identify people with high body temperature and stop them from travelling to reduce local transmissions. However, these infrared thermometers measure body surface temperature, which is not always a reliable surrogate for the core body temperature that is affected by infection. ${ }^{20}$ Infrared thermal cameras were usually mounted on a wall or ceiling to capture thermograms of the travelers' faces. ${ }^{21}$ These cameras were not used on a daily basis but only at the time of epidemics or pandemics. ${ }^{22,23}$ Several studies examined airport screening to evaluate the effectiveness of the entry/exit screening along with the reliability of the thermograms. ${ }^{13,18,19}$

\subsection{Infrared Thermography}

IRT, a non-contact and real-time thermometer, has become widely used in various clinical applications including oncology, dermatology, vascular disorders, and for fever screening. ${ }^{24-26}$ For example, inflammation from skin defects such as tungiasis was screened and quantified using IRT to measure inflammation-induced changes in skin temperature. ${ }^{24}$ In addition, thermal patterns of diabetic patients with and without vascular complications were compared using IRT. ${ }^{27}$ Unlike traditional thermographic instruments, IRT provides a live thermal map over a wide anatomical region, which enables analysis of body temperature distribution including any hot or cold spots. ${ }^{28}$ As a demonstration, Fig. 2 shows a thermal face map of an individual with and without fever.

IRT uses the properties of human infrared emission to capture thermographic information. All objects with a non-zero Kelvin temperature emit infrared radiation at wavelengths between 0.75 and $1000 \mu \mathrm{m}$. Human skin emissions range from 2 to $20 \mu \mathrm{m}$ in wavelength, peaking around $10 \mu \mathrm{m}$. The intensity of this infrared radiation can be mathematically translated to surface body temperature, a clinically important parameter. ${ }^{24,25,30}$ Together with the advantages of being a non-contact and real-time sensing technique with a wide anatomical region measurement, IRT emits no harmful radiation, which makes it suitable for public and long-term use. ${ }^{31}$ However, it requires a controlled environment, in which ambient temperature and humidity are

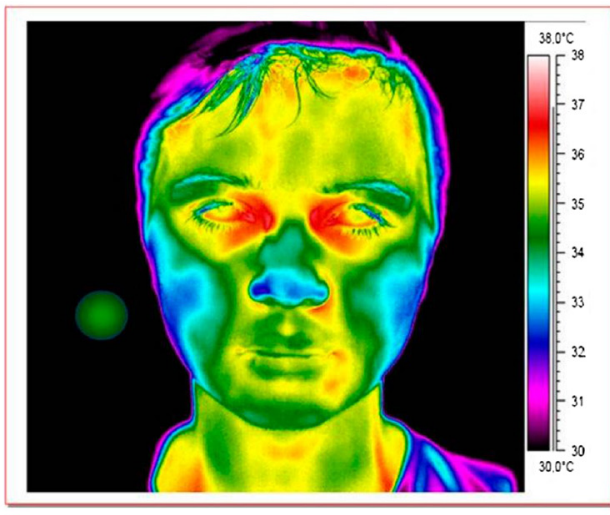

(a)

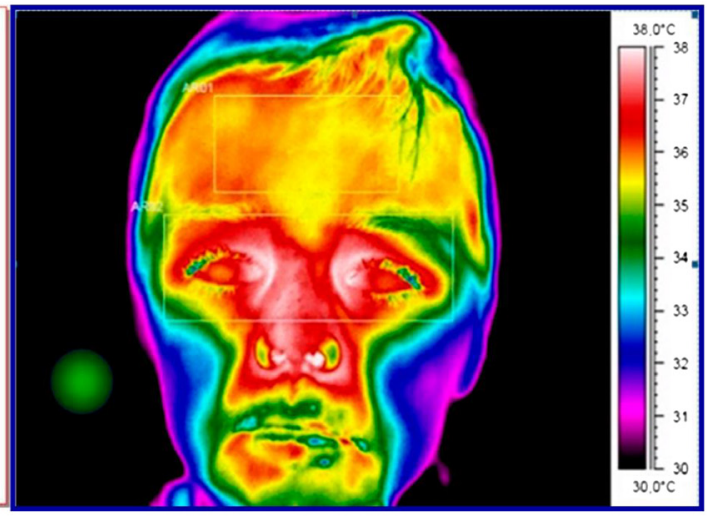

(b)

Fig. 2 Thermal image: (a) without fever and (b) with fever. ${ }^{29}$ 
maintained within specific ranges. Wide variance of such conditions can result in inaccurate thermographic readings. ${ }^{26}$ Additionally, IRT-based thermal screening is affected by other factors including medication, cosmetics, pregnancy, and physical activity. ${ }^{25,32}$

\subsubsection{IRT: instrumentation}

First generation thermal camera systems, developed in the 1970s, used a single infrared detector and two scanning mirrors to digitally generate a thermal image. Second generation thermal imagers, developed in the $1980 \mathrm{~s}$, introduced larger arrays of detectors (up to $64 \times 64$ ). ${ }^{32}$ These systems used time-delay integration for higher image quality. ${ }^{33}$ Current systems are comprised of an infrared sensor, image analysis hardware, and a real-time display monitor. ${ }^{25}$ Images captured by the infrared camera are converted into electrical signals and processed using a workstation or on-chip image processing hardware for real-time display and analysis. ${ }^{30}$ Upgrades to infrared sensor and camera technology have allowed for much larger two-dimensional detection arrays, upward of $1024 \times 1024$ elements, and improved optomechanical technology has permitted for the scanning of larger anatomical regions. ${ }^{30,33}$ The advance of microbolometric detectors, which require no cooling, has allowed for smaller and more lightweight IRT systems that can start up quickly. These advancements have also resulted in a significant improvement in noise equivalent temperature difference (NETD) of IRT systems over the last 20 years. Older IRT systems reported NETD in the 70- to 150 -mK range, whereas current systems, including compact low-cost technologies, report NETD of $<50 \mathrm{mK} .^{30,34,35}$

\subsubsection{IRT: data analysis and image processing}

IRT data processing involves digitizing the measured signal from the infrared camera, processing the data, and extracting body temperature. Digitization includes the transformation and quantification of infrared radiation into a spatial infrared image. These steps are dependent on the type of detector used, detector array size, and sampling rate of the system. ${ }^{31}$ Several pre- and postprocessing algorithms have been implemented in IRT systems to improve image quality. IR image preprocessing improves uniformity within images by correcting for fluctuating light conditions. ${ }^{36}$ Filters have been applied to thermal images to minimize noise and reduce blurring. In addition, operations including background subtraction and time derivative calculation of thermal data have been used to increase the accuracy of data. ${ }^{25}$ Current research to improve IRT data processing includes asymmetry analysis of thermal images, smart image enhancement and restoration algorithms, and automatic feature detection and classification algorithms. ${ }^{36}$ Effectively and efficiently detecting and segmenting anomalies in thermal images can be difficult to do with the sheer number of co-founding factors that can reduce the accuracy of the IRT images. Hierarchical clustering-based segmentation (HCS) is one such method of identifying features within IRT images that can be quite noisy. ${ }^{37}$ The advantage of this process compared to other segmentation algorithms is that HCS employs a hierarchy of thresholding rather than a single threshold value when identifying boundaries of regions of interests within IRT images. ${ }^{37}$ This minimizes the loss of useful data during the processing stage of IRT images. HCS is a versatile segmentation process that can be applied to IRT images from a wide variety of sources with minimal tuning. ${ }^{37-39}$ Examples include organic materials like plants in the environment and variations in body temperature postsurgery or inorganic cases such as differentiating different types of window materials using IRT imaging. To extract a temperature from processed infrared data, the physiological target radiation must be isolated from total radiation received by the camera, which also includes radiation from the atmosphere and surroundings. ${ }^{31}$

\subsubsection{Effects of environmental conditions on IRT results}

Several studies have been conducted to investigate the effects of various experimental parameters on thermal measurement accuracy. The United States Food and Drug Administration ${ }^{40}$ quantitatively compared two moderately priced commercial IRTs in various environmental conditions. The study found that the temperature, humidity, and type of data processing methods significantly affected IRT results. ${ }^{26}$ Ring et al. ${ }^{23}$ noted that the laboratory environment must be 
thermally stable. Research has also shown that factors such as system stability and drift, curvature of the anatomical region, precision of the IRT system, secondary infrared light sources, and participant use of cosmetics and antiperspirants can affect the accuracy of collected data. ${ }^{25,26}$

\subsubsection{State-of-the-art commercial and research systems}

Many commercial and research IRT systems have been developed. Commercial manufacturers include FLIR Systems Inc., which has produced numerous infrared thermal cameras with varying resolution, portability, and analysis capabilities. Seek thermal has created miniaturized, lowpower IRT systems including ones that can be attached to a smartphone or tablet camera, transforming it into a thermal imager. Table 2 lists specifications of two typical IR cameras, which have been used in many studies. ${ }^{41,42}$ Research systems adapted traditional IRT techniques and combined them with other clinical sensing modalities with software-based analysis tools. Several high-resolution IRT systems have been developed, including a three-dimensional IRT system in 2017 that can simultaneously obtain true-color images of the physiological region. ${ }^{24,43}$ A computer-assisted video thermography system has been developed that reduces subjectivity in interpretation of thermographic images by analyzing them using a software-based algorithm. ${ }^{44}$ In addition, a multi-modal system has been developed using IRT, a blood-flow meter, and microwave radar to provide more robust screening of suspected respiratory infection patients. ${ }^{45}$

\section{Materials and Methods}

A literature review was performed in Google Scholar, PubMed, and ScienceDirect to search for studies evaluating IRT screening from 2002 to present. The following key words were utilized: mass screening; fever; fever screening, thermometer, digital thermometer, IRT, thermal camera, thermogram, IRT sensitivity and specificity, IRT instrumentation, IRT data analysis, IRT generations, IRT components, infectious disease, respiratory infection, respiratory symptoms, infectious disease diagnosis, infectious disease detection, flu, COVID-19, SARS, MERS, Ebola, Influenza, Dengue, Zika, The Black Death, Smallpox, and HIV. The identified studies for the evaluation of mass screening using IRT were then selected based on the availability of information addressing total number of screened individuals, detected individuals, patients, device sensitivity, and specificity. Reported studies not containing the listed information were excluded from the review. Additional literature searches sought to identify literature specifically evaluating IRT in comparison to traditional measurements of core body temperature and comparing different IRT device components and instrumentation to each other through device sensitivity and specificity measurements. Finally, a search was done to locate studies assessing the benefit

Table 2 Specifications of two typical IR cameras.

\begin{tabular}{lcc}
\hline \hline Manufacturer & FLIR Systems, Inc. ${ }^{41}$ & Optotherm $^{42}$ \\
\hline Product & T500 series & Thermoscreen \\
Detector type & Uncooled microbolometer & Uncooled amorphous silicon \\
Pixel pitch $(\mu \mathrm{m})$ & 17 & 17 \\
Array size & $464 \times 348$ & $640 \times 480$ \\
Thermal sensitivity/NETD & $<30 \mathrm{mK}$ at $30^{\circ} \mathrm{C}$ & $<40 \mathrm{mK}^{\text {at }} 30^{\circ} \mathrm{C}$ \\
Accuracy & $\pm 2{ }^{\circ} \mathrm{C}$ & $\pm 0.3^{\circ} \mathrm{C} \mathrm{between} 30$ and $40^{\circ} \mathrm{C}$ \\
& & $\pm 1^{\circ} \mathrm{C}$ otherwise \\
Operating temperature range & $-15^{\circ} \mathrm{C}$ to $50^{\circ} \mathrm{C}$ & $15^{\circ} \mathrm{C}$ to $35^{\circ} \mathrm{C}$ \\
Frame rate $(\mathrm{Hz})$ & 30 & 60 \\
Working F-number & 1.1 with $42-\mathrm{deg}$ lens & 1.02 \\
\hline \hline
\end{tabular}


of implementing measurements of extra vital signs for the detection of infectious diseases from 2002 to present. The key words utilized were similar to the above list with inclusion of heart rate and breathing rate. Of the identified papers, studies reporting the number of subjects (patients and controls), device sensitivity, device specificity, PPV, and NPV were included. The identified literature not reporting these values were excluded.

For literature evaluating the effectiveness of IRT, six studies were identified comparing the measurements of surface and core body temperature for the detection of illness: one aimed at assessing wireless dermal thermometers as a replacement for invasive measurements, ${ }^{46}$ four studies aimed at studying the variations in skin temperature to variables unrelated to illness, ${ }^{20,47-49}$ and one analyzing the correlation between tympanic membrane temperature and the temperature at various facial regions. ${ }^{50,51}$ Additionally, four studies were found that investigated IRT accuracy using the forehead for the thermographic region of interest: one analyzing IRT for mass blind screening in Singapore, ${ }^{52}$ one assessing IRT during the H1N1 pandemic in Hong Kong, ${ }^{53}$ one from Taiwan assessing digital infrared thermal imaging (DITI) to conduct screenings on SARS patients, ${ }^{30}$ and one study in France evaluating IRT accuracy for fever screening. ${ }^{54}$ One study in the United States was found comparing the capabilities of three different infrared thermal detection systems and was included in the review. ${ }^{34}$ For literature assessing the locations for mass fever screenings, eight articles were identified; two aimed at the analysis of airport fever screening for Dengue in Taiwan; ${ }^{12,13}$ two aimed at fever screening at sea ports in Australia and Singapore; ${ }^{14,15}$ and two aimed at fever screening at border crossings in Singapore. ${ }^{15,16}$ Two studies were focusing on the effectiveness of IRT in screening COVID19 patients in Pakistan and United States. ${ }^{55,56}$ Additionally, three studies were identified assessing the effectiveness of screening to delay local transmission. ${ }^{17-19}$ Several studies were also included that analyzed the impact of a controlled environment on screening accuracy. ${ }^{21,57-59}$ For literature evaluating the efficiency of IRT screening for the detection of febrile international travelers, seventeen studies were identified: one aimed at detecting SARS in Canada ${ }^{60}$ four studies for Dengue detection in Taiwan; ${ }^{12,13,61,62}$ five for Influenza in New Zealand, ${ }^{63,64}$ Japan, ${ }^{65,66}$ and Australia; ${ }^{67}$ and three for Ebola virus disease (EVD) in the USA, Australia, and the UK, ${ }^{68}$ and Sierra Leone; ${ }^{69,70}$ one for MERS in Indonesia $;{ }^{71}$ and three for COVID-19 in multiple countries. ${ }^{72-74}$ For literature evaluating the detection of infectious diseases with measurements of vital signs, three studies were identified containing the required information for inclusion: two utilizing CMOS camera that was equipped with IRT, ${ }^{75,76}$ two utilizing Doppler blood-flow meter, $10-\mathrm{GHz}$ microwave radar, and thermography; $;^{77,78}$ and one utilizing radar, finger-tip photoreflector, and thermography. ${ }^{79}$

\section{Results}

\subsection{Specificity and Sensitivity of IRT in Detecting Fever}

Several studies have been conducted to investigate the efficacy of IRT as a tool for fever detection, which used the forehead as the thermographic region of interest. The results of these studies are summarized in Table 3. A 2004 study in Singapore by $\mathrm{Ng}$ et al. ${ }^{52}$ analyzed the capability of IRT for mass blind fever screening of 310 individuals and found a sensitivity and specificity of $89.6 \%$ and $94.3 \%$, respectively. They concluded that IRT can serve as the first line tool for fever screening if calibrated for outdoor environmental factors. A 2005 study in Hong Kong by $\mathrm{Ng}$ et al. compared non-contact infrared forehead temperature (NIFT) measurement to tympanic temperatures in 500 children. The study found that NIFT had a sensitivity and specificity of $89.4 \%$ and $75.4 \%$, respectively, of detecting fever using the cutoff point determined by tympanic temperature measurement. ${ }^{53}$ In Taiwan, Chiu et al. ${ }^{30}$ used a DITI system to conduct mass screening of suspected SARS patients. A total of 993 suspected febrile patients were screened and the study found a sensitivity and specificity of $75 \%$ and $99.6 \%$, respectively. A 2008 study in France evaluated the diagnostic accuracy of IRT for fever screening and tested 2026 patients in different groups based on predicted tympanic temperature. Sensitivity and specificity of their device were found to be $82 \%$ and $77 \%$, respectively. ${ }^{54}$ In the United States, Nguyen et al. ${ }^{34}$ compared three different infrared thermal detection systems, the FLIR ThermoVision, A20M, the Opto Therm 
Table 3 Summary of studies using IRT to detect fever.

\begin{tabular}{|c|c|c|c|}
\hline Author, year, country & $\begin{array}{l}\text { Sample } \\
\text { size }\end{array}$ & $\begin{array}{c}\text { Sensitivity } \\
(\%)\end{array}$ & $\begin{array}{l}\text { Specificity } \\
(\%)\end{array}$ \\
\hline $\mathrm{Ng}$ et al., 2004, Singapore ${ }^{52}$ & 310 & 89.6 & 94.3 \\
\hline Ng et al., 2005, Hong Kong ${ }^{53}$ & 500 & 89.4 & 75.4 \\
\hline Chiu et al., 2005, Taiwan ${ }^{30}$ & 993 & 75.0 & 99.6 \\
\hline Hausfater et al., 2008, France ${ }^{54}$ & 2026 & 82 & 77 \\
\hline \multirow{3}{*}{$\begin{array}{l}\text { Nguyen et al., 2010, United States (OptoTherm Thermoscreen, } \\
\text { FLIR ThermoVision A20M, Wahl Fever Alert Imager HSI20000S) }\end{array}$} & 2507 & 91.0 & 86.0 \\
\hline & 2515 & 90.0 & 80.0 \\
\hline & 2061 & 80.0 & 65.0 \\
\hline Khan et al., 2020, Pakistan ${ }^{55}$ & 538 & 13.61 & 97.95 \\
\hline \multirow{2}{*}{$\begin{array}{l}\text { Zhou et al. 2020, United States (A325sc, FLIR Systems Inc., } \\
8640 \text { P-series, Infrared Cameras Inc.) }\end{array}$} & 544 & 85 & 94 \\
\hline & 540 & 94 & 89 \\
\hline
\end{tabular}

Thermoscreen, and the Wahl Fever Alert Imager HSI20000S, to assess their screening capabilities. More than 2000 patients were tested with each system. The sensitivity and specificity of each device were as follows: FLIR $(90.0 \%, 80.0 \%)$, OptoTherm $(91.0 \%, 86.0 \%)$, and Wahl $(80.0 \%, 65.0 \%) .{ }^{34}$ In total, these values range from $75.0 \%$ to $91.0 \%$ for sensitivity and $65.0 \%$ to $99.6 \%$ for specificity. Variation in these values results from study-to-study differences in IRT device, experimental conditions, and threshold used to classify a successful measurement.

\subsection{Diseases and Public Screening by Infrared Thermography}

Entry/exit screening was performed during different pandemics including the influenza pandemic (H1N1) in 2009, MERS in Saudi Arabia in 2012, EVD in West Africa in 2014, SARS in Australia, Canada, and Singapore in 2003, and the most recent COVID-19 outbreak in China in $2019 .{ }^{63,64}$ However, very low sensitivity and specificity were reported. ${ }^{25}$ Health questionnaires, interviews, and careful examination of the traveler were suggested as alternatives because these provide a more extensive picture of the risk of someone having an infectious disease. ${ }^{80}$ Also social awareness, school closure, home quarantine, and social distancing are reported as more important variables in the disease transmission than entry/exit screening. ${ }^{81}$

Cowling et al., after the influenza type A (H1N1 or swine flu) outbreak, stated that entry screening of travelers may lead to short-term delay (1 to 2 weeks) in local transmission of influenza virus. In that work, they considered and reported on the results of 35 nations with more than $100 \mathrm{H} 1 \mathrm{~N} 1$ positive cases reported to the World Health Organization. ${ }^{81}$ In 2017, Sun et al. ${ }^{82}$ performed an IRT evaluation for detecting febrile international travelers entering Japan at Nagoya Airport (2003 to 2004) and Naha International Airport (2005 to 2009) after the SARS pandemic. They reported several limitations with the accuracy of IRT, such as taking antifebrile medications that affect the efficiency of IRT with a rapid modification of the body temperature.

\subsection{Mass, Blind Screening: Sensitivity and Specificity}

After the SARS pandemic in 2003, many countries established a mass screening system, usually a non-contact thermography system to detect fever in international airports. These systems have been employed to screen passengers at the entry and/or exit gate to prevent entry of the virus into a country and/or spreading the virus to other countries. Seventeen studies were identified that reported the efficiency of the screening system in detecting SARS (one study), dengue (four studies), influenza (five studies), EVD (three studies), MERS (one study), and COVID-19 (three 
studies). The summary of the total screened passenger, detected fever, detected patients, not detected patients, sensitivity, specificity, PPV, and NPV of these studies is presented in Table 4. The detection sensitivity was as low as 0\% in SARS, Ebola, influenza, MERS, and COVID-19 detections but was higher in Dengue, Chikungunya, and Zika detection.

Table 4 Mass screening of infectious disease in the airport.

\begin{tabular}{|c|c|c|c|c|c|c|c|c|c|}
\hline Country & Disease, year & Total & Detected & Patient & $\begin{array}{c}\text { Patient } \\
\text { not } \\
\text { detected }\end{array}$ & $\begin{array}{c}\text { Sensitivity } \\
(\%)\end{array}$ & $\begin{array}{c}\text { Specificity } \\
(\%)\end{array}$ & $\begin{array}{l}\text { PPV } \\
(\%)\end{array}$ & $\begin{array}{l}\text { NPV } \\
(\%)\end{array}$ \\
\hline Canada $^{60}$ & SARS, 2003 & $1,172,986$ & 2889 & 0 & - & 0 & - & - & - \\
\hline Taiwan $^{13}$ & $\begin{array}{l}\text { Dengue, } 2003 \\
\text { to } 2004\end{array}$ & $8,000,000$ & 22,000 & 40 & 25 & 65.8 & - & - & - \\
\hline Taiwan $^{61}$ & $\begin{array}{l}\text { Dengue, } 2003 \\
\text { to } 2007\end{array}$ & - & - & 244 & 298 & 45 & - & $\begin{array}{c}30.5 \text { to } \\
62.6\end{array}$ & - \\
\hline \multirow[t]{4}{*}{ Taiwan $^{12}$} & Dengue, 2007 & $12,508,621$ & 11,118 & 72 & 107 & 40.2 & 99.96 & 1.28 & 100 \\
\hline & 2008 & $12,202,392$ & 12,158 & 100 & 125 & 44.4 & 99.96 & 2.03 & 100 \\
\hline & 2009 & $12,499,365$ & 12,286 & 108 & 95 & 53.2 & 99.97 & 2.9 & 100 \\
\hline & 2010 & $14,837,391$ & 12,553 & 126 & 175 & 41.86 & 99.97 & 3.22 & 100 \\
\hline $\begin{array}{l}\text { New } \\
\text { Zealand }^{63}\end{array}$ & Influenza, 2008 & 5274 & 1275 & 30 & - & $\begin{array}{c}83 \text { to } \\
87\end{array}$ & $\begin{array}{c}11 \text { to } \\
39\end{array}$ & $\begin{array}{c}2.0 \text { to } \\
2.8\end{array}$ & - \\
\hline Australia $^{67}$ & $\begin{array}{l}\text { H1N1 Influenza, } \\
2009\end{array}$ & 625,147 & 5845 & 3 & 45 & 6.67 & 99.1 & 0.05 & - \\
\hline $\begin{array}{l}\text { New } \\
\text { Zealand }\end{array}$ & $\begin{array}{l}\text { H1N1 Influenza, } \\
2009\end{array}$ & 456,518 & 406 & 4 & 69 & 5.80 & - & - & - \\
\hline Japan $^{65}$ & $\begin{array}{l}\text { H1N1 Influenza, } \\
2009\end{array}$ & 471,733 & 805 & 10 & 141 & 6.60 & - & - & - \\
\hline$J^{J a p a n}{ }^{66}$ & $\begin{array}{l}\text { H1N1 Influenza, } \\
2009 \text { to } 2010\end{array}$ & $9,140,435$ & 930 & 0 & - & 0 & - & - & - \\
\hline \multirow[t]{9}{*}{ Taiwan $^{62}$} & Dengue, 2013 & $19,072,276$ & 12,924 & 115 & 148 & 44 & - & - & - \\
\hline & Chikungunya & & & 17 & 29 & 59 & - & - & - \\
\hline & Dengue & $21,707,379$ & 15,280 & 118 & 127 & 48 & - & - & - \\
\hline & Chikungunya & & & 4 & 7 & 57 & - & - & 一 \\
\hline & Dengue & $23,601,215$ & 17,779 & 155 & 210 & 42 & - & - & 一 \\
\hline & Chikungunya & & & 4 & 0 & 100 & - & - & 一 \\
\hline & Zika & $21,083,404$ & 21,721 & 5 & 8 & 38 & - & - & 一 \\
\hline & Dengue & & & 130 & 185 & 41 & - & - & 一 \\
\hline & Chikungunya & & & 4 & 4 & 50 & - & - & 一 \\
\hline US $^{68}$ & Ebola, 2014 & 1993 & 86 & 0 & - & 0 & - & - & - \\
\hline Australia $^{68}$ & & 122 & 6 & 0 & - & 0 & - & - & - \\
\hline$U^{68}$ & & 3388 & 130 & 0 & - & 0 & - & - & - \\
\hline
\end{tabular}


Table 4 (Continued).

\begin{tabular}{|c|c|c|c|c|c|c|c|c|c|}
\hline Country & Disease, year & Total & Detected & Patient & $\begin{array}{c}\text { Patient } \\
\text { not } \\
\text { detected }\end{array}$ & $\begin{array}{c}\text { Sensitivity } \\
(\%)\end{array}$ & $\begin{array}{c}\text { Specificity } \\
(\%)\end{array}$ & $\begin{array}{l}\text { PPV } \\
(\%)\end{array}$ & $\begin{array}{l}\text { NPV } \\
(\%)\end{array}$ \\
\hline $\begin{array}{l}\text { Guinea, } \\
\text { Liberia, } \\
\text { and Sierra } \\
\text { Leone }^{69}\end{array}$ & $\begin{array}{l}\text { Ebola, } 2014 \\
\text { to } 2016\end{array}$ & 300,000 & - & 0 & 4 & 0 & - & - & - \\
\hline $\begin{array}{l}\text { Sierra } \\
\text { Leone }^{70}\end{array}$ & $\begin{array}{l}\text { EVD, } 2014 \\
\text { to } 2016\end{array}$ & 166,242 & 10 & 0 & 2 & 0 & - & - & - \\
\hline Indonesia $^{71}$ & MERS, 2015 & 28,197 & 15 & 0 & - & 0 & - & - & - \\
\hline India 72 & $\begin{array}{l}\text { COVID-19, } \\
2020\end{array}$ & $1,587,034$ & 151 & 0 & - & 0 & - & - & - \\
\hline $\begin{array}{l}\text { Multiple } \\
\text { countries }^{73}\end{array}$ & $\begin{array}{l}\text { COVID-19, } \\
2020\end{array}$ & - & - & 14 & 257 & 5.2 & & & \\
\hline US $^{74}$ & $\begin{array}{l}\text { COVID-19, } \\
2020\end{array}$ & 766,044 & 278 & 9 & 14 & 39 & & & \\
\hline
\end{tabular}

\subsection{Combination of IRT with Other Techniques for Screening Infectious Disease}

Along with temperature, several groups have suggested the measurement of extra vital signs including heart rate and respiratory rate. ${ }^{75-78}$ They have claimed that since the inflammation not only causes an elevation in body temperature but also increases to heart and respiration rates, inclusion of these multiple vital signs will improve screening accuracy. In 2010, Matsui et al. ${ }^{75}$ employed laser doppler-flow meter to obtain heart rate, $10-\mathrm{GHz}$ microwave radar to detect breathing rate, and thermography to measure skin temperature on 92 subjects ( 57 patients with $\mathrm{H} 1 \mathrm{~N} 1$ influenza and 35 controls). By applying linear discriminant analysis on the multimodal data, they achieved $88 \%$ sensitivity and $89 \%$ specificity. After that, Yao et al. ${ }^{78}$ used other classification algorithms, including support vector machine, $k$-nearest neighbors, and logistic regression, on the same data sets to improve the sensitivity to $93 \%$. Similarly, high sensitivity $(97.1 \%$ and $87.5 \%)$ and specificity $(81.3 \%$ and $100 \%)$ were obtained in other studies when multimodal signals were measured. ${ }^{77-79}$ Notably, Sun et al. claimed that the inclusion of heart rate and respiration rate enhances the sensitivity by $18.8 \%$ compared to when temperature alone was used. Table 5 summarizes the techniques used, number of subjects, sensitivity, specificity, PPV, and

Table 5 Summary of five studies using different techniques to measure heart rate, respiration rate, and temperature in patient with influenza and healthy controls.

\begin{tabular}{|c|c|c|c|c|c|c|c|c|}
\hline \multirow[b]{2}{*}{ Paper } & \multirow[b]{2}{*}{ System } & \multicolumn{2}{|c|}{$\begin{array}{l}\text { Number of } \\
\text { subjects }\end{array}$} & \multicolumn{2}{|c|}{ Sensitivity (\%) } & \multirow[b]{2}{*}{$\begin{array}{c}\text { Specificity } \\
(\%)\end{array}$} & \multirow[b]{2}{*}{$\begin{array}{l}\text { PPV } \\
(\%)\end{array}$} & \multirow[b]{2}{*}{$\begin{array}{l}\text { NPV } \\
(\%)\end{array}$} \\
\hline & & Patient & Control & $\begin{array}{l}\text { Fever- } \\
\text { based }\end{array}$ & All & & & \\
\hline Sun, $2017^{75}$ & $\begin{array}{l}\text { CMOS camera equipped } \\
\text { with IRT }\end{array}$ & 16 & 22 & 68.70 & 87.50 & 100 & 100 & 91.70 \\
\hline Negishi, $2019^{76}$ & & 12 & 13 & - & - & - & - & - \\
\hline Matsui, $2010^{77}$ & $\begin{array}{l}\text { Doppler blood-flow meter, } \\
10-\mathrm{GHz} \text { microwave radar, }\end{array}$ & 57 & 35 & - & 88 & 89 & 93 & 82 \\
\hline Yao, $2016^{78}$ & thermography & 57 & 35 & - & 93 & - & - & - \\
\hline Sun, $2015^{79}$ & $\begin{array}{l}\text { Radar, finger-tip } \\
\text { photoreflector, } \\
\text { thermography }\end{array}$ & 35 & 48 & - & 97.10 & 81.30 & 79.10 & 97.50 \\
\hline
\end{tabular}


NPV of five studies measuring multiple vital signs to classify patients with influenza from the healthy control.

\section{Discussion}

Though IRT is capable of real-time, non-contact measurement of body surface temperature over a wide anatomical area, its measurement accuracy depends heavily on environmental parameters. Additionally, body surface temperature is not always a reliable surrogate for the core body temperature that is affected by infection. Rectal and esophageal temperature are reported to be the most reliable and easily accessible body sites to obtain core body temperature ${ }^{21}$ but they are invasive and not appropriate sites for mass screening. On the other hand, sublingual, axillary, inguinal sites, auditory canal, and forehead are more common sites for measuring temperature using clinical thermometers, but they do not reflect the true core body temperature. In this regard, modern IRT suggests capturing thermograms of the human face non-invasively and using various algorithms to compensate for the underestimated core body temperature. Here the question would be which site(s) on the human face is the best representative of the change in core temperature. Ring et al ${ }^{83}$ suggested using canthus measurement as a more reliable measurement of the core temperature. Although it is not difficult to detect an increase in body temperature through canthi, there is a complicated relationship between this temperature and the real core temperature. Other studies focused on forehead or auditory meatus temperature for easier measurement, but limitations are reported. ${ }^{84}$ Ultimately, the lack of scientific data showing the relationship between human head (face) temperature and core body temperature remains a challenge to be addressed.

Another challenge with mass screening using IRT is the inability to detect the fever development in incubating or asymptomatic patients during early or late stages. An infected individual might not present with a fever during the incubation period. Thus the febrile screening system is not able to capture the case. Additionally, a normal body temperature will be for previously infected individual who are already on fever suppressant. On the other hand, there might be other conditions for a reported high fever that are not due to a viral infection. Some medications such as hormone treatments, severe trauma and injury, and other medical conditions or pregnancy can cause an elevated body temperature. In these cases, a reported high fever with IRT might be mistaken as an infection.

The presence of a fever due to an infection depends on various parameters such as age, the immune system status, the inflicting virus, and the disease stage. A study in Finland examined patients with laboratory-confirmed influenza and found fever present in $89 \%$ of children younger than 13 years old ${ }^{85}$ In another work, Chughtai et al ${ }^{86}$ stated that fever is less common in adults with confirmed respiratory infections than in children, reporting that $75 \%$ of adults showed no fever. Carrat et al. compared fever in adults with different types of influenza and found that the prevalence of fever in those inflicted with influenza differs between viral strains (H3N2: 95.2\%, H1N1: 77.5\%). ${ }^{87}$ Moreover, it is reported that some infectious respiratory diseases have only respiratory symptoms. ${ }^{88}$

Camera quality plays an important role in thermography. Low camera resolution, poor focus, and placing the camera too far from the subject may cause inaccurate measurement. Training personnel, regularly testing cameras, and following essential protocols may help with reliability and reproducibility of the outcomes of the technique. ${ }^{21}$

Although fever is a primary symptom in the majority of infectious diseases, many studies have demonstrated that measuring body temperature alone is insufficient in detecting infections. ${ }^{12,13,60-70,75-79}$ As has been suggested previously, in addition to body temperature, heart rate and respiratory rate are the two crucial vital signs needed to be monitored. ${ }^{75-79}$ Additionally, since many infectious diseases, especially coronavirus related infections, cause SARS, monitoring breathing related parameters may enhance sensitivity and specificity of disease screening.

\section{Conclusion}

The COVID-19 outbreak and resultant efforts in preventing disease transmission has raised the alarm to re-examine screening methods for infectious diseases. High temperature, a typical 
indicator of an infection, is the only parameter considered for mass screenings at airports and borders during an epidemic. Since the 2003 SARS outbreak, infrared thermal cameras have been mounted at airports in countries such as Canada, Taiwan, and Australia. ${ }^{13,60,68}$ Several groups studied the efficacy of mass fever screening using thermal cameras in those airports, but they have not found reliable outcomes in detecting febrile individuals using these systems. Low camera quality, very low sensitivity to true body temperature, and inability to detect asymptomatic patients were reported as the main reasons that thermal cameras alone are not reliable. ${ }^{21}$ It has been suggested that other vital physiological parameters should be monitored as extra indicators of an infection to obtain more consistent results from mass screening. ${ }^{75-79}$ In addition to an elevated body temperature, a patient with respiratory infectious disease such as COVID-19 experiences a change in tissue oxygenation, cardiovascular, and respiratory functions. Therefore, there is an urgent need to develop a new technique capable of rapidly screening all these signals and integrating the measured parameters into new metrics for early detection of viral infections. With the advent of wireless technologies, this approach, ideally, can lead to the development of sensors with point-of-care home-accessible capabilities to manage the growing number of infected patients staying in home quarantine, eventually alleviating the burden on the healthcare system.

\section{Disclosures}

The authors declare no conflict of interest.

\section{Acknowledgments}

This study was supported by intramural research program of Eunice Kennedy Shrive National Institute of Child Health and Human Development of the National Institutes of Health. Babak Shadgan holds a Scholar Award from the Michael Smith Foundation for Health Research.

\section{References}

1. H. Li, Y. Zhou, and Z. Zhang, "Network analysis reveals a common host-pathogen interaction pattern in Arabidopsis immune responses," Front. Plant Sci. 8, 893 (2017).

2. S. N. DeWitte, "Mortality risk and survival in the aftermath of the medieval Black Death," PLoS One 9, e96513 (2014).

3. D. Huremović, "Brief history of pandemics (Pandemics throughout history)," in Psychiatry of Pandemics, D. Huremović, Ed., Springer, Cham, Switzerland (2019).

4. Y. Yi et al., "COVID-19: what has been learned and to be learned about the novel coronavirus disease," Int. J. Biol. Sci. 16, 1753-1766 (2020).

5. Johns Hopkins Hospital and Medicine, Coronavirus COVID-19 Global Cases by the Center for Systems Science and Engineering (CSSE) at Johns Hopkins University (JHU), Johns Hopkins University.

6. CDC, Interim Laboratory Biosafety Guidelines for Handling and Processing Specimens Associated with Coronavirus Disease 2019 (COVID-19), Centers for Disease Control and Prevention (2020).

7. D. Ogoina, "Fever, fever patterns and diseases called "fever": a review," J. Infect. Public Health 4, 108-124 (2011).

8. S. Banoo et al., "Evaluation of diagnostic tests for infectious diseases: general principles," Nat. Rev. Microbiol. 4, S21-S31 (2010).

9. J. A. Al-Tawfiq and P. G. Auwaerter, "Healthcare-associated infections: the hallmark of Middle East respiratory syndrome coronavirus with review of the literature," J. Hosp. Infect. 101, 20-29 (2019).

10. E. Livingston, A. Desai, and M. Berkwits, "Sourcing personal protective equipment during the COVID-19 pandemic," J. Am. Med. Assoc. 323, 1912-1914 (2020).

11. N. Chow et al., "Preliminary estimates of the prevalence of selected underlying health conditions among patients with coronavirus disease 2019-United States, February 12-March 28, 2020," Morb. Mortal. Wkly. Rep. 69, 382-386 (2020). 
12. M. M. Kuan and F. Y. Chang, "Airport sentinel surveillance and entry quarantine for dengue infections following a fever screening program in Taiwan," BMC Infect. Dis. 12, 1-10 (2012).

13. P. Y. Shu et al., "Fever screening at airports and imported dengue," Emerg. Infect. Dis. 11(3), 460-462 (2005).

14. G. Samaan et al., "Border screening for SARS in Australia: what has been learnt?" Med. J. Aust. 180, 220-223 (2004).

15. A. Wilder-Smith, N. I. Paton, and K. T. Goh, "Experience of severe acute respiratory syndrome in Singapore: importation of cases, and defense strategies at the airport," J. Travel Med. 10(5), 259-262 (2003).

16. C. C. Tan, "SARS in Singapore: key lessons from an epidemic," Ann. Acad. Med. Singapore 35(5), 345-349 (2006).

17. V. A. Mouchtouri et al., "Exit and entry screening practices for infectious diseases among travelers at points of entry: looking for evidence on public health impact," Int. J. Environ. Res. Public Health 16(23), 4638 (2019).

18. L. A. Selvey, C. Antão, and R. Hall, "Entry screening for infectious diseases in humans," Emerg. Infect. Dis. 21, 197-201 (2015).

19. Y. L. Huizer et al., "Usefulness and applicability of infectious disease control measures in air travel: a review," Travel Med. Infect. Dis. 13(1), 19-30 (2015).

20. C. Huizenga et al., "Skin and core temperature response to partial- and whole-body heating and cooling," J. Thermal Biol. 29, 549-558 (2004).

21. J. B. Mercer and E. F. J. Ring, "Fever screening and infrared thermal imaging: concerns and guidelines," Thermol. Int. 19(3), 67-69 (2009).

22. G. Sengoz et al., "Prepared pandemic plan for H1N1 in Istanbul metropolis, April-June 2009: procedures and first results," Clin. Microbiol. Infect. (2010).

23. E. F. J. Ring, "ISO standards for fever screening and their implications," Thermol. Int. (2012).

24. A. Schuster et al., "High-resolution infrared thermography: a new tool to assess tungiasisassociated inflammation of the skin," Trop. Med. Health 45, 23 (2017).

25. B. B. Lahiri et al., "Medical applications of infrared thermography: a review," Infrared Phys. Technol. 55, 221-235 (2012).

26. P. Ghassemi et al., "Best practices for standardized performance testing of infrared thermographs intended for fever screening," PLoS One 13(9), e0203302 (2018).

27. F. Ring, "Thermal imaging today and its relevance to diabetes," J. Diabetes Sci. Technol. 4, 857-862 (2010).

28. A. Di Carlo, "Thermography and the possibilities for its applications in clinical and experimental dermatology," Clin. Dermatol. 13(4), 329-336 (1995).

29. F. Ring, "The Herschel heritage to medical thermography," J. Imaging 2, 13 (2016).

30. W. T. Chiu et al., "Infrared thermography to mass-screen suspected SARS patients with fever," Asia-Pac. J. Public Health 17, 26-28 (2005).

31. R. Usamentiaga et al., "Infrared thermography for temperature measurement and nondestructive testing," Sensors (Switzerland) 14, 12305-12348 (2014).

32. D. Bitar, A. Goubar, and J. C. Desenclos, "International travels and fever screening during epidemics: a literature review on the effectiveness and potential use of non-contact infrared thermometers," Euro Surveill. 14, 19115 (2009).

33. H. Qi and N. Diakides, "Infrared imaging in medicine," Kiv (2007).

34. A. V. Nguyen et al., "Comparison of 3 infrared thermal detection systems and self-report for mass fever screening," Emerg. Infect. Dis. 16(11), 1710-1717 (2010).

35. E. Villa et al., "Performance assessment of low-cost thermal cameras for medical applications," Sensors 20(5), 1321 (2020).

36. O. Faust et al., "Application of infrared thermography in computer aided diagnosis," Infrared Phys. Technol. 66, 160-175 (2014).

37. A. N. Selvan and C. Childs, "Infrared thermal mapping, analysis and interpretation in biomedicine," (2017).

38. A. D. A. N. Selvan, "Hierarchical clustering-based segmentation (HCS) aided diagstic image interpretation monitoring," $\mathrm{PhD}$ Thesis, Sheffield Hallam University, United Kingdom, PQDT-Glob. (2007). 
39. C. Childs et al., "Thermal territories of the abdomen after caesarean section birth: infrared thermography and analysis," J. Wound Care 25, 499-512 (2016).

40. M. Hillen et al., "Cluster analysis of IR thermography data for differentiating glass types in historical leaded-glass windows," Appl. Sci. 10, 4255 (2020).

41. Flir Systems, "FLIR T500 series spec sheet," https://www.flir.com/globalassets/importedassets/document/17-0881-t500-series-datasheet.pdf (2020).

42. Optotherm, "Optotherm thermoscreen data sheet," https://www.optotherm.com/ Thermoscreen\%20Brochure\%20Jan\%202020.pdf (2020).

43. V. S. Cheng, J. Bai, and Y. Chen, "A high-resolution three-dimensional far-infrared thermal and true-color imaging system for medical applications. Med. Eng. Phys. 31, 1173-1181 (2009).

44. F. J. P. M. Huygen et al., "Computer-assisted skin videothermography is a highly sensitive quality tool in the diagnosis and monitoring of complex regional pain syndrome type 1," Eur. J. Appl. Physiol. 91, 516-524 (2004).

45. T. Matsui et al., "Development of a non-contact screening system for rapid medical inspection at a quarantine depot using a laser Doppler blood-flow meter, microwave radar and infrared thermography," J. Med. Eng. Technol. 33, 481-487 (2009).

46. H. Zsuzsanna Balla, E. Theodorsson, and J. O. Ström, "Evaluation of commercial, wireless dermal thermometers for surrogate measurements of core temperature," Scand. J. Clin. Lab. Invest. 79, 1-6 (2019).

47. K. G. Ng et al., "Evaluation of the Cadi ThermoSENSOR wireless skin-contact thermometer against ear and axillary temperatures in children," J. Pediatr. Nurs. 25(3), 176-186 (2010).

48. G. Booth, "Changes in temperature of the skin following the ingestion of food," Arch. Intern. Med. 57(3), 533 (1936).

49. K. Pathak et al., "Forearm to fingertip skin temperature gradients in the thermoneutral zone were significantly related to resting metabolic rate: potential implications for nutrition research," Eur. J. Clin. Nutr. 71, 1074-1079 (2017).

50. D. W. Kim et al., "The correlation between tympanic membrane temperature and specific region of face temperature," Quantum Infrared Thermogr. J. 16(1), 1-7 (2019).

51. D. W. Kim et al., "The correlation between tympanic membrane temperature and specific region of face temperature," (2017).

52. E. Y. K. Ng, G. J. L. Kawb, and W. M. Chang, "Analysis of IR thermal imager for mass blind fever screening," Microvasc. Res. 68(2), 104-109 (2004).

53. D. K. Ng et al., "Non-contact infrared thermometry temperature measurement for screening fever in children," Ann. Trop. Paediatr. 25, 267-275 (2005).

54. P. Hausfater et al., "Cutaneous infrared thermometry for detecting febrile patients," Emerg. Infect. Dis. 14, 1255-1258 (2008).

55. N. S. Khan et al., "Usefulness of forehead infrared thermometers to screen patients for fever during COVID-19 Pandemic," Pakistan Armed Forces Med. J. (2020).

56. Y. Zhou et al., "Clinical evaluation of fever-screening thermography: impact of consensus guidelines and facial measurement location," J. Biomed. Opt. 25, 097002 (2020).

57. T. Suzuki et al., "The validity of mass body temperature screening with ear thermometers in a warm thermal environment," Tohoku J. Exp. Med. 222, 89-95 (2010).

58. P. Ghassemi et al., "Standardized assessment of infrared thermographic fever screening system performance," Proc. SPIE 10056, 100560H (2017).

59. K. D. Lafferty, "The ecology of climate change and infectious diseases," Ecology 90, 888-900 (2009).

60. R. K. St. John et al., "Border screening for SARS," Emerg. Infect. Dis. 11, 6-10 (2005).

61. M. M. Kuan et al., "Epidemiological trends and the effect of airport fever screening on prevention of domestic dengue fever outbreaks in Taiwan, 1998-2007," Int. J. Infect. Dis. 14, e693-e697 (2010).

62. L. L. Ho et al., "Taiwan's travel and border health measures in response to Zika," Health Secur. 15, 185-191 (2017).

63. P. C. Priest et al., "Thermal image scanning for influenza border screening: results of an airport screening study," PLoS One. 6, e14490 (2011). 
64. M. J. Hale, R. S. Hoskins, and M. G. Baker, "Screening for influenza A(H1N1)pdm09, Auckland International Airport, New Zealand," Emerg. Infect. Dis. 18(5), 866-868 (2012).

65. H. Sakaguchi et al., "Assessment of border control measures and community containment measures used in Japan during the early stages of Pandemic (H1N1) 2009," PLoS One 7, e31289 (2012).

66. H. Nishiura and K. Kamiya, "Fever screening during the influenza (H1N1-2009) pandemic at Narita International Airport, Japan," BMC Infect Dis. 11, 111 (2011).

67. P. J. Gunaratnam et al., "Airport arrivals screening during pandemic (H1N1) 2009 influenza in New South Wales, Australia," Med. J. Aust. 200, 290-292 (2014).

68. C. M. Brown et al., "Airport exit and entry screening for Ebola-August-November 10, 2014," Morb. Mortal Wkly. Rep. 63, 1163-1167 (2014).

69. N. J. Cohen et al., "Travel and border health measures to prevent the international spread of Ebola," Morb. Mortal Wkly. Rep. Suppl. 65, 57-67 (2016).

70. K. Wickramage, "Airport entry and exit screening during the Ebola virus disease outbreak in Sierra Leone, 2014 to 2016," Biomed. Res. Int. 2019, 1-6 (2019).

71. M. Amin et al., "Screening for Middle East respiratory syndrome coronavirus among febrile Indonesian Hajj pilgrims: a study on 28,197 returning pilgrims," J. Infect. Prev. 19, 236-239 (2018).

72. A. R. Pasi et al., "Early detection of suspected cases of COVID-19: role of thermal screening at international airports in India," Int. J. Commun. Med. Public Health (2020).

73. V. A. Mouchtouri et al., "Detection of imported COVID-19 cases worldwide: early assessment of airport entry screening, 24 January until 17 February 2020," Trop. Med. Health 48, 79 (2020).

74. P. Dollard et al., "Risk assessment and management of COVID-19 among travelers arriving at designated U.S. Airports, January 17-September 13, 2020," Morb. Mortal. Wkly. Rep. (2020).

75. G. Sun et al., "Remote sensing of multiple vital signs using a CMOS camera-equipped infrared thermography system and its clinical application in rapidly screening patients with suspected infectious diseases," Int. J. Infect. Dis. 55, 113-117 (2017).

76. T. Negishi et al., "Infection screening system using thermography and CCD camera with good stability and swiftness for non-contact vital-signs measurement by feature matching and MUSIC algorithm," in Proc. Annu. Int. Conf. IEEE Eng. Med. and Biol. Soc., EMBS (2019).

77. T. Matsui et al., "A novel screening method for influenza patients using a newly developed non-contact screening system," J. Infect. 60, 271-277 (2010).

78. Y. Yao et al., "Multiple vital-sign-based infection screening outperforms thermography independent of the classification algorithm," IEEE Trans. Biomed. Eng. 63, 1025-1033 (2016).

79. G. Sun et al., "An infectious disease/fever screening radar system which stratifies higherrisk patients within ten seconds using a neural network and the fuzzy grouping method," J. Infect. 70, 230-236 (2015).

80. Canadian Agency for Drugs and Technologies in Health, "Non-contact thermometers for detecting fever: a review of clinical effectiveness," CADTH Rapid Response Reports (2014).

81. B. J. Cowling et al., "Entry screening to delay local transmission of 2009 pandemic influenza A (H1N1)," BMC Infect. Dis. 10, 8-11 (2010).

82. G. Sun et al., "Applications of infrared thermography for noncontact and noninvasive mass screening of febrile international travelers at airport quarantine stations," (2017).

83. E. F. J. Ring et al., "New standards for fever screening with thermal imaging systems," J. Mech. Med. Biol. 13, 1350045 (2013).

84. C.-C. Liu, R.-E. Chang, and W.-C. Chang, "Limitations of forehead infrared body temperature detection for fever screening for severe acute respiratory syndrome," Infect. Control Hosp. Epidemiol. 25, 1109-1111 (2004).

85. S. Heinonen et al., "Signs and symptoms predicting influenza in children: a matched casecontrol analysis of prospectively collected clinical data," Eur. J. Clin. Microbiol. Infect. Dis. 31, 1569-1574 (2012). 
86. A. A. Chughtai et al., "The presence of fever in adults with influenza and other viral respiratory infections," Epidemiol. Infect. 145, 148-155 (2017).

87. F. Carrat et al., "Evaluation of clinical case definitions of influenza: detailed investigation of patients during the 1995-1996 epidemic in France," Clin. Infect. Dis. 28, 283-290 (1999).

88. J. P. Ridgway et al., "Influenza among afebrile and vaccinated healthcare workers," Clin. Infect. Dis. 60, 1591-1595 (2015).

Kosar Khaksari received her BS degree in biomedical engineering from Amirkabir University of Technology (Tehran Polytechnic) in 2007, her MS degree from the Department of Electrical and Computer Engineering at the University of Tehran, and her MS and PhD degrees in biomedical engineering from Michigan Technological University in 2016. She is a postdoctoral IRTA at the National Institutes of Health. Her main research interests are using optical techniques to look at blood flow, perfusion, and tissue oxygenation.

Thien Nguyen received her BS degree in biomedical engineering from Hanoi University of Science and Technology in 2013, and her MS and PhD degrees in biomedical science and engineering from Gwangju Institute of Science and Technology in 2015 and 2019, respectively. She is currently a postdoctoral fellow at the National Institutes of Health. She is an author of more than 20 journals and conference papers. Her current research interests include optical applications in brain activity and tissue oxygenation.

Babak Shadgan is a an assistant professor in the Department of Orthopaedics at the University of British Columbia (UBC), an associate faculty member at the UBC School of Biomedical Engineering and a principal investigator at the International Collaboration on Repair Discoveries (ICORD), where he is directing the Clinical Biophotonics Laboratory. With more than two decades of medical practice and research, he has developed a specific knowledge in clinical applications of biosensing technologies with a unique integrated transitional bedside-tobench and bench-to-bedside approach. He is actively working on design and development of novel wearable and implantable biosensors and their applications in health and diseases. He is a fellow member, an instructor, and a conference chair at the International Society for Optics and Photonics (SPIE).

Amir H. Gandjbakhche received his $\mathrm{PhD}$ in physics with a biomedical engineering specialty from the University of Paris in 1989. He is a senior investigator at the National Institutes of Health and the head of the Section on Analytical and Functional Biophotonics of NICHD. $\mathrm{He}$ is a fellow of SPIE. He leads a research group that uses different optical sources of contrast to develop quantitative theories at the board, designs instrumentation at the bench, and brings the imaging system to the bedside. His areas of interest are the use of near-infrared spectroscopy/ EEG to apply to the developmental disorders and diseases such as cognitive function in traumatic brain injury and autistic spectrum disorder and using spectroscopic methods to quantify oxygenation in placenta.

Biographies of the other authors are not available. 